\title{
Meconium-stained Amniotic Fluid Revisited: A Holistic Perspective
}

\author{
Rajesh Panicker ${ }^{1}$, Lei Lei Win², Jaipal Moopil ${ }^{3}$
}

\begin{abstract}
Since historical times, the presence of meconium in the amniotic fluid has been worrisome for midwives and accoucheurs alike. Its association with a neonate who does not cry has often been a chill factor in delivery suites. Having said that, all cases of meconium-stained amniotic fluid (MSAF) do not necessarily result in low APGAR scores. In addition to in utero fetal hypoxia, meconium passage has also been associated with maternal drug abuse, use of vaginal misoprostol for induction of labor, chorioamnionitis, and maternal diabetes. The main pathology associated with MSAF is the aspiration of meconium during intrauterine gasping or during the first few breaths. This causes meconium aspiration syndrome (MAS) which has serious consequences on neonatal outcome. MAS is a common cause of severe respiratory distress in term neonates, with an associated highly variable morbidity and mortality. The pathophysiology of MAS is multifactorial and includes acute airway obstruction, surfactant dysfunction or inactivation, chemical pneumonitis, and persistent pulmonary hypertension of a newborn. Concepts regarding meconium and the management of MSAF to prevent MAS have changed in the last two decades or so. Guidelines published by the American Academy of Pediatrics/American Heart Association have changed the immediate neonatal management following delivery in the presence of MSAF. Initially, amnioinfusion was considered an important tool in the management of MSAF. However, evidence to support this view has not been forthcoming and current guidelines recommend amnioinfusion only in controlled and research settings. The future thrust should be aimed at early detection of MSAF and prevention of MAS. Needless to say, the obstetrician and the neonatologist need to work in consonance for achieving a better neonatal outcome in the presence of MSAF.

Keywords: Aspiration, Chill factor, Meconium, Perinatal mortality, Perinatal outcome, Pregnancy, Prevention.

Journal of South Asian Federation of Obstetrics and Gynaecology (2019): 10.5005/jp-journals-10006-1658
\end{abstract}

Aristotle was believed to have observed the tranquilizing effect of meconium on the newborn which probably explains the etymology of meconium from the Greek mēkōnion meaning the juice of the opium poppy. ${ }^{1} \mathrm{MSAF}$ is seen in $8-20 \%$ of all deliveries and may increase to $23-52 \%$ after 42 weeks of gestation which has been attributed to the gastrointestinal maturation. ${ }^{2,3}$ MSAF is more common among women of African and Asian ethnicity. ${ }^{4}$

Meconium is a dark-green substance which contains gastrointestinal secretions, amniotic fluid, bile acids, bile pigments, blood, mucus, cholesterol, pancreatic secretions, lanugo, vernix caseosa, and cellular debris. ${ }^{5}$ Meconium is the first intestinal discharge following delivery and it is believed that it accumulates within the fetal gastrointestinal tract throughout the third trimester of pregnancy. It is usually released within the first 48 hours after birth.

MSAF continues to be a chill factor in delivery suites primarily because of the association with fetal hypoxia and poor APGAR scores which is why the attending staff scurry around for help at the time of delivery. ${ }^{6,7}$ Though MSAF has been considered as a marker for fetal hypoxia, this association between fetal distress and in utero passage of meconium has not been conclusively substantiated. Meconium passage has also been associated with maternal drug abuse, use of vaginal misoprostol for induction of labor, chorioamnionitis, and maternal diabetes. ${ }^{8}$ Apart from these associations, very little is known about in utero passage of meconium. Since the process of labor and delivery are stressful to the fetus, it is possible that this stress could result in biochemical events that result in the passage of meconium during the normal course of labor.

The main pathology associated with MSAF is the aspiration of meconium during intrauterine gasping or during the first few
1,3 Department of Obstetrics and Gynaecology, AIMST University, Bedong, Malaysia

${ }^{2}$ Department of Paediatrics, AIMST University, Bedong, Malaysia

Corresponding Author: Rajesh Panicker, Department of Obstetrics and Gynaecology, AIMST University, Bedong, Malaysia, Phone: +60 1126146711, e-mail: rpanicker@rediffmail.com

How to cite this article: Panicker R, Win LL, et al. Meconium-stained Amniotic Fluid Revisited: A Holistic Perspective. J South Asian Feder Obst Gynae 2019;11(2):131-133.

Source of support: Nil

Conflict of interest: None

breaths. This causes MAS which has serious consequences on neonatal outcome.

\section{What's New and What's Changed}

Meconium has been considered to be sterile. However, researchers have isolated bacterial remnants and communities within meconium. These are mainly of two types, namely those bacteria that produce lactic acid, such as lactobacillus, and those that belong to the family of the so-called enteric bacteria, such as Escherichia coli. ${ }^{9}$ Needless to say, this has clinical significance. In one study of the singleton pregnancies that ultimately had a cesarean delivery after a trial of labor, 5,883 had MSAF and those with MSAF had a significantly increased incidence of surgical site infection. ${ }^{10}$

The mechanisms underlying meconium passage in utero are not clear and appear to be complex. As mentioned before, the association of MSAF with fetal distress has been known since historic times. The explanation could lie in increased parasympathetic activity causing increased intestinal peristalsis and relaxation of the 
anal sphincter secondary to enhanced vagal output during episodes of fetal hypoxia. A study has also shown that the corticotropinreleasing factor, a known mediator of colonic motility, has been implicated in the pathogenesis of hypoxia-induced MSAF in a rat model. ${ }^{11}$ Having said that, can we conclusively state that the presence of MSAF is a reliable sign of fetal hypoxia? The nuanced answer to that is that the current evidence does show an association between MSAF and fetal hypoxia, but are all cases of MSAF a reliable indicator of acute fetal distress, the answer has to be no.

Clinicians have associated the consistency and time of passage of meconium with adverse fetal outcomes. The risk of perinatal death is increased five to seven times when freshly passed or thick meconium is present at the onset of labor. ${ }^{12}$ Infants with thin or old meconium are more likely to have passed meconium as a physiologic maturational process and they are more likely to be healthy at birth. ${ }^{13}$ Moreover, there is evidence that MSAF was associated with an increase in the rate of pathological intrapartum fetal heart rate patterns, intrapartum fevers, and operative vaginal and cesarean section deliveries, and these complications increased with the staining and thickness of the amniotic fluid. ${ }^{14}$ As a consequence of this, the consistency of meconium was an important factor in determining whether a neonate needs immediate endotracheal suctioning at birth. However, current evidence no longer supports this practice. If the infant is vigorous with good respiratory effort and a heart rate $>100$ beats/minutes, tracheal intubation to aspirate meconium should not be attempted; only the mouth and nose may be suctioned with a bulb or suction catheter. If the neonate is depressed, traditional teaching has always been that in the presence of MSAF, any form of positive pressure ventilation should be preceded by endotracheal suctioning or else it could result in the meconium being pushed further into the air passages. However, the current American Academy of Pediatrics/ American Heart Association guidelines no longer recommend endotracheal suctioning before positive pressure ventilation even in depressed infants (2015 guidelines). ${ }^{15}$

Perineal suctioning of the oropharynx was recommended in the past in the presence of MSAF. However, based on available evidence, routine perineal suctioning of all babies in the presence of MSAF does not prevent MAS and has the potential risk of delaying proper resuscitative measures. ${ }^{13,16}$

In the past, transcervical amnioscopy has been performed to visualize the color of the membranes, to predict the presence of MSAF. This was considered as a means of antenatal assessment of fetal wellbeing. However, this procedure is invasive and is no longer performed in a clinical setting, as meconium staining is no longer considered to be a reliable sign of acute fetal hypoxia.

Amnioinfusion, or transcervical infusion of normal saline, at body temperature, into the amniotic cavity, has been proposed as a method of reducing the risk of the MAS in patients with MSAF. The potential mechanisms include dilution of meconium, thus, reducing its mechanical and inflammatory effects, and cushioning of the umbilical cord, thus, correcting recurrent umbilical-cord compressions that lead to variable fetal heart rate decelerations and fetal acidemia. However, it must be kept in mind that this procedure is not without risk and the complications include uterine overdistension, hypertonus, infection, and amniotic fluid embolism. According to the ACOG Committee report 2006 (reaffirmed in 2018), the current evidence does not show that amnioinfusion reduces the incidence of MAS in patients with MSAF. NICE guidance of Nov 2006 also states that amnioinfusion should not be used routinely as a therapeutic tool and may be used only in a research setting.
Can ultrasonogram (USG) be used to predict the presence of meconium in the amniotic fluid? There is some evidence to show that echogenic amniotic fluid is associated with an increased incidence of MSAF; however, the sensitivity of this investigative modality appears to be poor. ${ }^{17}$

\section{Meconium Aspiration Syndrom}

Meconium aspiration syndrom (MAS) has been defined as respiratory distress in an infant born through meconium-stained amniotic fluid with radiologic changes suggestive of this syndrome and whose symptoms cannot be otherwise explained. ${ }^{18}$ It is a common cause of severe respiratory distress in term neonates, with an associated highly variable morbidity and mortality. The pathophysiology of MAS is multifactorial and includes acute airway obstruction, surfactant dysfunction or inactivation, chemical pneumonitis, and persistent pulmonary hypertension of a newborn. ${ }^{18}$ Short-term complications include death, respiratory failure, ventilator-induced barotrauma, and development of pulmonary hypertension. Long-term complications include pulmonary sequelae such as wheezing in infancy and asthma in childhood. Long-term complications also include adverse neurodevelopmental outcomes.

This disorder is often life threatening, and approaches to the prevention of MAS have changed over time with collaboration between obstetricians and pediatricians forming the foundations for care. The wide use of surfactant and inhaled nitric oxide as therapeutic tools has led to decreased mortality and the need for extracorporeal membrane oxygenation. ${ }^{18}$ In developed countries, mortality rates have been reduced to below $5 \% .{ }^{19}$

Approximately $30-50 \%$ of infants diagnosed with MAS will require continuous positive airway pressure (CPAP) or mechanical ventilation and these conventional ventilatory methods still remain the mainstay in the management of MAS. The optimum modes of ventilation for MAS, however, are yet to be formulated. ${ }^{20}$

Does cesarean section reduce the incidence of MAS in patients with MSAF? This question has troubled both pediatricians and obstetricians. However, there is no evidence to show a difference in the neurodevelopmental outcomes of surviving infants born by normal spontaneous vaginal delivery vs cesarean section delivery. ${ }^{21}$

\section{Future Research and Guidance}

Divon et al. evaluated the National Swedish Medical Birth Registry. In 181,524 singleton pregnancies with reliable dates delivered at greater than 40 weeks, the authors found a significant increase in the odds ratio for fetal death, due to MAS, at greater than 41 weeks of gestation. ${ }^{22}$ Hence, there could be grounds for deliberations on the current definition of postterm pregnancy which still remains 42 weeks or more.

Research in the area of prevention and treatment of MAS is ongoing especially on the role and timing of surfactant therapy and the role of antibiotics. Studies are also being conducted to determine "best" ventilatory strategies for the different grades of MAS. ${ }^{20}$

A device to visualize the amniotic fluid prior to the rupture of membranes was tried out in the past without much success. ${ }^{23}$ This is one area where technology and high-resolution ultrasound can be focused to provide a noninvasive method of detecting MSAF in the future.

\section{ConCLUSiOn}

In conclusion, we can say that the problem of MSAF will be faced by clinicians and the best way to reduce the "chill factor" would be 
to understand the pathogenesis of meconium release and its effect on the fetal respiratory system, and to be prepared to manage MAS. Multimodal management involving the obstetrician and the neonatologist would be the ideal approach to this problem. Strategies for the prevention of MSAF and MAS need to be formulated and standardized by consultative cooperation between the neonatologist and the obstetrician.

\section{References}

1. Vain NE, Batton DG. "Meconium "Aspiration". Semin Fetal Neonatal Med 2017;22(4):214-219. DOI: 10.1016/j.siny.2017.04.002.

2. Cleary GM, Wiswell TE. Meconium-stained amniotic fluid and the meconium aspiration syndrome: an update. Pediatr Clin North Am 1998;45(3):511-529. DOI: 10.1016/S0031-3955(05)70025-0.

3. Ostrea EM, Jr, Naqvi M. The influence of gestational age on the ability of the fetus to pass meconium in utero: clinical implications. Acta Obstet Gynecol Scand 1982;61(3):275-277. DOI: 10.3109/00016348209156571.

4. Balchin I, Whittaker JC, et al. Maternal and fetal characteristics associated with meconium-stained amniotic fluid. Obstet Gynecol 2011;117(4):828-835. DOI: 10.1097/AOG.0b013e3182117a26.

5. Van lerland Y, De Beaufort AJ. "Why Does Meconium Cause Meconium Aspiration Syndrome? Current Concepts of MAS Pathophysiology". Early Hum Dev 2009;85:617-620. DOI: 10.1016/ j.earlhumdev.2009.09.009.

6. Dargaville PA, Copnell B, et al. The epidemiology of meconium aspiration syndrome: incidence, risk factors, therapies, and outcome. Pediatrics 2006;117(5):1712-1721. DOI: 10.1542/peds.2005-2215.

7. Pramanik AK, Rengaswamy N, et al. Neonatal respiratory distress: a practical approach to its diagnosis and management. Pediatr Clin North Am 2015;62(2):453-469. DOI: 10.1016/j.pcl.2014.11.008.

8. Unsworth J. Meconium in labour. Obstet Gynaecol Reprod Med 2010;20(10):289-294. DOI: 10.1016/j.ogrm.2010.06.005.

9. Jiménez $E$, Marín $M L$, et al. Is meconium from healthy newborns actually sterile? Res Micro 2008;159(3):187-193. DOI: 10.1016/ j.resmic.2007.12.007.

10. Snyder A, Rood KM, et al. Meconium-Stained Amniotic Fluid is Associated with an Increased Risk of Post-Cesarean Surgical Site Infection. Obstet Gynecol May 2017;129(5):1S. DOI: 10.1080/ 14767058.2019.1637408.

11. Lakshmanan J, Ahanya SN, et al. Elevated plasma corticotrophin release factor levels and in utero meconium passage. Pediatr Res 2007;61:176. DOI: 10.1203/pdr.0b013e31802d8a81.
12. Rossi EM, Philipson EH, et al. Meconium aspiration syndrome: intrapartum and neonatal attributes. Am J Obstet Gynecol 1989;161:1106-1110. DOI: 10.1016/0002-9378(89)90643-1.

13. Wiswell TE, Bent RC. Meconium staining and the meconium aspiration syndrome. Unresolved issues. Pediatr Clin North Am 1993;40:955-981. DOI: 10.1016/S0031-3955(16)38618-7.

14. Rodríguez Fernández V, Ramón Y Cajal CNL, et al. Intrapartum and perinatal results associated with different degrees of staining of meconium stained amniotic fluid. Eur J Obstet Gynecol Reprod Biol 2018 Sep;228:65-70. DOI: 10.1016/j.ejogrb.2018.03.035.

15. American Heart Association: Part 13: Neonatal Resuscitation. Web-based Integrated 2010 and 2015 American Heart Association Guidelines for Neonatal Cardiopulmonary Resuscitation and Emergency Cardiovascular Care. AHA website. Updated 2015. https:// eccguidelines.heart.org/index.php/circulation/cpr-ecc-guidelines-2/ part-13-neonatal-resuscitation/.

16. Newton O, English M. Newborn resuscitation: defining best practice for low-income settings: transactions of Royal Society of Tropical Medicine and Hygene. Oct 2006;100(10-4):899-908. DOI: 10.1016/j. trstmh.2006.02.012.

17. Karamustafaoglu Balci B, Goynumer G. Incidence of echogenic amniotic fluid at term pregnancy and its association with meconium. Arch Gynecol Obstet 2018;297:915. DOI: s00404-018-4679-7.

18. Swarnam K, Soraisham AS, et al. Advances in the management of meconium aspiration syndrome. Int J Pediatr 2012;2012:359571. DOI: 10.1155/2012/359571.

19. Bhat $R$, Vidyasagar $D$. Delivery room management of meconiumstained infant. Clin Perinatol 2012;39(4):817-831. DOI: 10.1016/ j.clp.2012.09.004.

20. Goldsmith JP. Continuous positive airway pressure and conventional mechanical ventilation in the treatment of meconium aspiration syndrome. J Perinatol 2008;28(Suppl 3):S49-S55. DOI: 10.1038/ jp.2008.156.

21. Beligere N, Rao R. Neurodevelopmental outcome of infants with meconium aspiration syndrome: report of a study and literature review. J Perinatol 2008 Dec;28(Suppl 3):S93-S101. DOI: 10.1038/ jp.2008.154.

22. Divon MY, Haglund B, et al. Fetal and neonatal mortality in the postterm pregnancy: the impact of gestational age and fetal growth restriction. Am J Obstet Gynecol 1998;178:726. DOI: 10.1016/S00029378(98)70482-X.

23. Doody Michael C. Assignee. Prenatal detection of meconium stained amniotic fluid. US pat. US5172693. 1992 Dec 22. 